\title{
Extraordinary Temperature Dependence of the Resonant Andreev Reflection
}

\author{
Yu Zhu, Qing-feng Sun and Tsung-han Lin* \\ State Key Laboratory for Mesoscopic Physics and \\ Department of Physics, Peking University, Beijing \\ 100871, China
}

\begin{abstract}
An extraordinary temperature dependence of the resonant Andreev reflection via discrete energy level in a normal-metal / quantum-dot / superconductor (N-QD-S) system is predicted theoretically by using Green function technique. The width of zero bias conductance peak in N-QD-S is about $\sqrt{\Gamma_{L}^{2}+\Gamma_{R}^{2}}$ and does not exhibit thermal broadening, where $\Gamma_{L}$ and $\Gamma_{R}$ are the coupling strength between QD and leads. Considering the intra-dot Coulomb interaction, the Coulomb blockade oscillations conducted by Andreev reflection differs dramatically from that in N-QD-N. Instead of thermal broadening, finite temperature induces more resonant peaks around the oscillation peaks of zero temperature. This effect can be applied to determine the coupling strength and QD level spacing in N-QD-S.

PACS numbers: 74.50.+r, 73.40.Gk, 73.20.Dx, 72.15.Nj.
\end{abstract}


Mesoscopic hybrid normal-metal / superconductor (N/S) systems have been investigated intensively in the last decade. Aside from their potential applications, these systems provide us an opportunity to combine with the two different quantum coherent behaviors, the coherence of quasi-particles in mesoscopic system and the coherence Cooper pairs in superconductors. The key mechanism to connect the above two together is the Andreev reflection (AR) process at the N/S interface [1, []. Many new phenomena and effects involving AR have been addressed in recent years (for a review see [3] and references therein). Among them, normal-metal / quantum-dot / superconductor (N-QD-S) is one of the interesting systems, in which resonant AR occurs via discrete energy levels of QD [⿴囗大 6 [6]. Considering the strong intra-dot interaction, the problem of Kondo resonance in N-QD-S were also studied by several authors [7-11]. In this paper, we shall predict the unique temperature dependence of the resonant AR process and investigate the interplay of Coulomb blockade effect and the Andreev tunneling in the N-QD-S systems.

Temperature usually gives us the impression of averaging and smearing. At finite temperature, the sharp resonant conductance peak usually has a thermal broadening of the order of $k_{B} T$, but we find this is not true for the case of resonant $\mathrm{AR}$ conductance. Let us begin by considering a N-QD-S system modeled by the following Hamiltonian,

$$
\begin{aligned}
H & =H_{L}+H_{R}+H_{d o t}+H_{T}, \\
H_{L} & =\sum_{k \sigma} \epsilon_{k} a_{k \sigma}^{\dagger} a_{k \sigma}, \\
H_{R} & =\sum_{p \sigma} \epsilon_{p} b_{p \sigma}^{\dagger} b_{p \sigma}+\sum_{p}\left(\Delta b_{p \uparrow}^{\dagger} b_{-p \downarrow}^{\dagger}+h . c\right), \\
H_{d o t} & =\sum_{\sigma} E_{0} c_{\sigma}^{\dagger} c_{\sigma}, \\
H_{T} & =\sum_{k \sigma}\left(t_{L} a_{k \sigma}^{\dagger} c_{\sigma}+\text { h.c }\right)+\sum_{p \sigma}\left(t_{R} b_{p \sigma}^{\dagger} c_{\sigma}+\text { h.c }\right),
\end{aligned}
$$

where $H_{L}$ and $H_{R}$ describe the left $\mathrm{N}$ lead and the right $\mathrm{S}$ lead, $H_{\text {dot }}$ describes QD with one spin-degenerate energy level, and $H_{T}$ is the tunneling between them.

In the regime of $k_{B} T \ll \Delta$, the zero bias conductance is dominated by the AR process, and can be derived by using Green function technique (see [6]) as,

$$
G_{N D S}=\frac{4 e^{2}}{h} \int d \omega\left[-\frac{\partial f(\omega)}{\partial \omega}\right] \Gamma_{L}^{2}\left|G_{12}^{r}(\omega)\right|^{2},
$$

where $f(\omega)$ is the Fermi distribution function, $\Gamma_{L}$ is the coupling strength (defined later), $G_{12}^{r}$ is the 12 element of $2 \times 2 \mathrm{Nambu}$ matrix $\mathbf{G}^{r}$, depicting the conversion from an electron 
into a hole. $\mathbf{G}^{r}$ is defined by

$$
\mathrm{G}^{r}(\omega)=\int d t e^{-\mathrm{i} \omega t}(-\mathrm{i}) \theta(t)\left(\begin{array}{c}
<\left\{c_{\uparrow}(t), c_{\uparrow}^{\dagger}(0)\right\}><\left\{c_{\uparrow}(t), c_{\downarrow}(0)\right\}> \\
<\left\{c_{\downarrow}^{\dagger}(t), c_{\uparrow}^{\dagger}(0)\right\}><\left\{c_{\downarrow}^{\dagger}(t), c_{\downarrow}(0)\right\}>
\end{array}\right),
$$

and can be solved from the Dyson equation,

$$
\mathbf{G}^{r}=\mathbf{g}^{r}+\mathbf{g}^{r} \Sigma^{r} \mathbf{G}^{r}
$$

where

$$
\begin{gathered}
\mathbf{g}^{r}=\left(\begin{array}{cc}
\frac{1}{\omega-E_{0}+\mathrm{i} 0^{+}} & 0 \\
0 & \frac{1}{\omega+E_{0}+\mathrm{i} 0^{+}}
\end{array}\right), \\
\boldsymbol{\Sigma}^{r}=-\frac{\mathrm{i}}{2} \Gamma_{L}\left(\begin{array}{ll}
1 & 0 \\
0 & 1
\end{array}\right)-\frac{\mathrm{i}}{2} \Gamma_{R} \rho_{R}\left(\begin{array}{cc}
1 & -\frac{\Delta}{\omega} \\
-\frac{\Delta}{\omega} & 1
\end{array}\right),
\end{gathered}
$$

in which

$$
\rho_{R} \equiv \begin{cases}\frac{|\omega|}{\sqrt{\omega^{2}-\Delta^{2}}} & |\omega|>\Delta \\ \frac{\omega}{\mathrm{i} \sqrt{\Delta^{2}-\omega^{2}}} & |\omega|<\Delta\end{cases}
$$

and $\Gamma_{L / R} \equiv 2 \pi N_{L / R}\left|t_{L / R}\right|^{2}$, with $N_{L / R}$ being the density of states in the left/right lead in normal state. For comparison we also write down the zero bias conductance for N-QD-N |12],

$$
G_{N D N}=\frac{2 e^{2}}{h} \Gamma_{L} \Gamma_{R} \int d \omega\left[-\frac{\partial f(\omega)}{\partial \omega}\right] \frac{1}{\left(\omega-E_{0}\right)^{2}+\left(\frac{\Gamma_{L}+\Gamma_{R}}{2}\right)^{2}},
$$

in which single particle tunneling process dominates. In deriving the above formulas, we assume that $\Gamma_{L / R}$ is independent of $\omega$, which is reasonable in the calculation of zero bias conductance [12]. In the two limits of $k_{B} T \ll \Gamma_{L}, \Gamma_{R}$ and $k_{B} T \gg \Gamma_{L}, \Gamma_{R}$, the integrands of Eq.(2) and Eq.(8) can be evaluated, and the results are summarized in the following table

\begin{tabular}{|l|l|l|l|l|}
\hline system & londition & conductance & half peak width & peak shape \\
\hline N-QD-S & $k_{B} T \ll \Gamma_{L}, \Gamma_{R}$ & $4 \frac{e^{2}}{h} \frac{\Gamma_{L}^{2} \Gamma_{R}^{2}}{4\left(E_{0}^{2}+\frac{\Gamma_{L}^{2}+\Gamma_{R}^{2}}{4}\right)^{2}}$ & $0.64 \sqrt{\Gamma_{L}^{2}+\Gamma_{R}^{2}}$ & squared Lorentzian \\
\hline N-QD-S & $k_{B} T \gg \Gamma_{L}, \Gamma_{R}$ & $4 \frac{e^{2}}{h} \frac{\pi}{4 k_{B} T} \frac{\Gamma_{L} \Gamma_{R}^{2}}{4\left(E_{0}^{2}+\frac{\Gamma_{L}^{2}+\Gamma_{R}^{2}}{4}\right)}$ & $\sqrt{\Gamma_{L}^{2}+\Gamma_{R}^{2}}$ & Lorentzian \\
\hline N-QD-N & $k_{B} T \ll \Gamma_{L}, \Gamma_{R}$ & $2 \frac{e^{2}}{h} \frac{\Gamma_{L} \Gamma_{R}}{E_{0}^{2}+\left(\frac{\Gamma_{L}+\Gamma_{R}}{2}\right)^{2}}$ & $\Gamma_{L}+\Gamma_{R}$ & Lorentzian \\
\hline N-QD-N & $k_{B} T \gg \Gamma_{L}, \Gamma_{R}$ & $2 \frac{e^{2}}{h} \frac{\pi}{k_{B} T} \frac{e^{\beta E_{0}}}{\left(e^{\beta E_{0}+1}\right)^{2}} \frac{2 \Gamma_{L} \Gamma_{R}}{\Gamma_{L}+\Gamma_{R}}$ & $3.53 k_{B} T$ & derivative of $f(\omega)$ \\
\hline
\end{tabular}


Fig. 1 shows the curves of $G_{N D S}$ vs $E_{0}$ and $G_{N D N}$ vs $E_{0}$ at different temperatures for the symmetric coupling case $\Gamma_{L}=\Gamma_{R} \equiv \Gamma$. One can see the following features: (1) For $T \rightarrow 0$, $G_{N D S}$ has its maximum $4 e^{2} / h$ at $E_{0}=0$ while $G_{N D N}$ has $2 e^{2} / h$, and $G_{N D S}$ is much steeper than $G_{N D N}$ which is of the Lorentzian shape. (2) With the increase of temperature, both $G_{N D S}$ and $G_{N D N}$ are suppressed, but surprisingly, the conductance peak of $G_{N D S}$ does not exhibit thermal broadening while $G_{N D N}$ obviously does. Table (9) tells us that the peak width of $G_{N D S}$ is always of the order $\sqrt{\Gamma_{L}^{2}+\Gamma_{R}^{2}}$ for either $k_{B} T \ll \Gamma_{L}, \Gamma_{R}$ or $k_{B} T \gg \Gamma_{L}, \Gamma_{R}$. Feature (1) has been obtained in [4], while feature (2) is addressed for the first time in this work, to our knowledge.

Taking into account of that AR is a special two-particle process, feature (2) has a simple interpretation shown in Fig.2. For N-QD-N (Fig.2b), the conductance is dominated by the process of single-particle tunneling. At finite temperature, the Fermi surface of leads spreads out around the chemical potential in the range of $k_{B} T$, allowing incident electron has energy $E$ in this range. The resonant tunneling occurs when $E_{0}=E$, resulting in the broadening of conductance peak of the order $k_{B} T$. For N-QD-S (Fig.2a), on the contrast, the singleparticle tunneling is forbidden due to superconducting gap, and AR process dominates. In the AR process, an incident electron with energy $E$ (with respect to the superconductor chemical potential) picks up another electron with energy $-E$ to form Cooper pair and enter S, with a hole reflected in the Fermi sea of N. Since both the incident electron and the picked electron pass $\mathrm{QD}$ through the discrete energy level $E_{0}$, the resonant tunneling requires not only $E_{0}=E$ but also $E_{0}=-E$. Therefore, the resonant $\mathrm{AR}$ process occurs only when $E_{0}=0$, i.e., $E_{0}$ lines up with the chemical potential of superconductor. Because $E_{0}$ has a small broadening and shifting due to coupling with the leads, the condition $E_{0}=0$ becomes to $\left|E_{0}\right|<O(\Gamma)$ with $O(\Gamma)$ being the order of the coupling strength. Thus, the resonant AR causes a bottle-neck of the conductance peak width in N-QD-S, leading to a broadening about $\Gamma$ instead of $k_{B} T$.

Next, we investigate a more realistic model, in which QD has multiple single-particle energy levels and intra-dot Coulomb interaction, i.e.,

$$
H_{d o t}=\sum_{i \sigma} E_{i} c_{i \sigma}^{\dagger} c_{i \sigma}+\frac{U}{2} \sum_{i \alpha \neq j \beta} n_{i \alpha} n_{j \beta},
$$

where $i, j$ are indices of the single-particle energy levels, $\sigma, \alpha, \beta$ are indices of the spin, and $U$ is the strength of Coulomb interaction. For N-QD-N with intra-dot Coulomb interaction, it is well known that the zero bias conductance vs the gate voltage exhibits Coulomb blockade 
oscillations (CBO) [14]. For N-QD-S in which one of N electrodes is replaced by $\mathrm{S}$, we also expect to see CBO except the conducting mechanism is AR. Taking into account of the unusual temperature dependence of the resonant AR process, CBO in N-QD-S may have significant change from that in N-QD-N.

We constrain ourselves to discuss the weak coupling and low temperature regime, i.e., $\Gamma \ll k_{B} T \ll \Delta$. If the coupling between QD and leads is weak enough, the atomic limit solution $(\Gamma \rightarrow 0)$ 13 should be a good starting point. Because we study the zero bias conductance, i.e., $V_{L}-V_{R}=0^{+}$, QD can be viewed as in equilibrium, and the current driven by the small bias voltage servers as a probe to the state of interacting QD. By generalizing the exact solution of the retarded Green function of QD in the atomic limit (see the Appendix for details), we propose the following scheme for the calculation of zero bias conductance. Suppose QD has $l$ spin-degenerate energy levels, $E_{1 \uparrow}, E_{1 \downarrow}, E_{2 \uparrow}, E_{2 \downarrow}, \cdots E_{l \uparrow}, E_{l \downarrow}$, with $2^{2 l}$ configurations of occupation represented by $F=\left(N_{1 \uparrow}, N_{1 \downarrow}, N_{2 \uparrow}, N_{2 \downarrow}, \cdots N_{l \uparrow}, N_{l \downarrow}\right)$ in which $N_{i \sigma}=0$ or 1 . In the weak coupling regime, the retarded Green function of QD can be derived by an approximated Dyson equation

$$
\mathbf{G}^{r}=\tilde{\mathbf{g}}^{r}+\tilde{\mathbf{g}}^{r} \Sigma^{r} \mathbf{G}^{r}
$$

in which $\tilde{\mathbf{g}}^{r}$ is the atomic limit solution for an isolated interacting QD and $\boldsymbol{\Sigma}^{r}$ is the selfenergy caused by the tunneling between QD and leads. $\Sigma^{r}$ is presented in Eq. (6), and $\tilde{\mathbf{g}}^{r}$ can be obtained as

$$
\begin{aligned}
\tilde{\mathbf{g}}^{r} & \equiv \sum_{F} P(F) \mathbf{g}^{r}(F), \\
P(F) & =\frac{1}{Z} e^{-\beta E_{d o t}(F)}, Z=\sum_{F} e^{-\beta E_{d o t}(F)}, \\
\mathbf{g}^{r}(F) & =\left(\begin{array}{cc}
\sum_{i} \frac{1}{\omega-\tilde{E}_{i \uparrow}(F)+i 0^{+}} & 0 \\
0 & \sum_{i} \frac{1}{\omega+\tilde{E}_{i \downarrow}(F)+i 0^{+}}
\end{array}\right),
\end{aligned}
$$

in which $E_{d o t}(F) \equiv \sum_{i \sigma} E_{i \sigma} N_{i \sigma}+\frac{U}{2} \sum_{i \alpha \neq j \beta} N_{i \alpha} N_{j \beta}$ is the total energy of QD for the configuration $F$, and $\tilde{E}_{i \sigma}(F) \equiv E_{i \sigma}+U \sum_{j \beta \neq i \sigma} N_{j \beta}$ is the renormalized sing-particle levels of QD. And the total conductance through QD can be expressed in terms of $\mathbf{G}^{r}$ derived from the above equations [6].

We plot $G_{N D S}$ vs $V_{g}$ and $G_{N D N}$ vs $V_{g}$ for different temperatures in Fig.3a-3d and Fig.3e, where QD contains two spin-degenerate levels, $E_{1 \uparrow}=E_{1 \downarrow}=E_{1}=V_{g}, E_{2 \uparrow}=E_{2 \downarrow}=E_{2}=$ 
$V_{g}+\Delta E$, with level spacing $\Delta E=0.1$, interacting constant $U=1$. For $k_{B} T \ll \Delta E$, we see typical CBO pattern in Fig.3a, with nearly equal spaced peaks at $V_{g}=0.0,1.0,2.1,3.1$. With the increase of temperature, peak heights are suppressed, roughly proportional to $\frac{1}{k_{B} T}$ (Notice different scaling in Fig.3a-3d). The most striking feature of CBO in N-QD-S is, when $k_{B} T \sim \Delta E$, temperature induces more resonant peaks around the original CBO peaks, instead of thermal broadening. One can see in Fig.3a-3d that the separation among peak groups is about $U$, while the spacing of peaks within one peak group is $\frac{\Delta E}{2}$, and each resonant peak has a width about $\Gamma$. Notice that all these properties are independent on temperature.

Qualitatively, this unusual pattern can be understood as follows. Due to the intra-dot Coulomb interaction, the original two resonances $E_{1}=E_{1 \uparrow}=E_{1 \downarrow}$ and $E_{2}=E_{2 \uparrow}=E_{2 \downarrow}$ exhibits eight sub-resonances, $E_{1}, E_{2}, E_{1}+U, E_{2}+U, E_{1}+2 U, E_{2}+2 U, E_{1}+3 U, E_{2}+3 U$ (see the appendix and Ref. [15] for detail). At zero temperature, only four resonances $E_{1}, E_{1}+U, E_{2}+2 U, E_{2}+3 U$ are active, and the other four are Coulomb blockaded. But for higher temperature of $k_{B} T \sim \Delta E$, the Coulomb blockade effect is partially removed. Not only $E_{1}$ but also $E_{2}$ are active and contribute to the total conductance. For N-QD-N, since resonant peak is broadened by finite temperature, the contributions of $E_{1}$ and $E_{2}$ are indistinguishable and combined to form the first $\mathrm{CBO}$ peak around $V_{g}=0$ in Fig.3e. (This mechanism was first proposed by Y. Meir et al. to explain the anomalous temperature dependence of CBO peak heights in N-QD-N [15].) For N-QD-S, however, resonant AR peak has the width of $\Gamma$ instead of $k_{B} T$. The first group of peaks around $V_{g}=0$ in Fig.3c consists of three distinguishable contributions of AR: AR via $E_{1}$, AR via $E_{2}$, and AR between $E_{1}$ and $E_{2}$ [6]. Other groups of peaks can be understood similarly. Furthermore, one find groups with tiny peaks around $V_{g}=0.5,1.5,2.5$ in Fig.3d, which can be attributed to the AR process between $E_{1}$ and $E_{1}+U, E_{1}$ and $E_{2}+U, E_{2}$ and $E_{2}+U, E_{2}$ and $E_{1}+U$, etc. In short, temperature dependence of $\mathrm{CBO}$ in N-QD-S differs dramatically from that in N-QD-N.

Finally, we would like to make three remarks: (1) The extraordinary temperature dependence of $\mathrm{CBO}$ in N-QD-S provides a new approach to determine the coupling strength $\Gamma \sim \sqrt{\Gamma_{L}^{2}+\Gamma_{R}^{2}}$ and QD level spacing $\Delta E$, which are impossible to do in N-QD-N because of thermal broadening. (2) The suggested N-QD-S structure is accessible of the up-date nano-technology, either by a metal nano-particle connected to superconducting and normal metal electrodes [16, 17], or in a gate controlled N-2DEG-S structure [18 20]. The key point to observe the predicted unusual temperature dependence of AR is to perform resonant $\mathrm{AR}$ 
process via discrete energy levels. (3) Similar extraordinary temperature dependence of zero bias conductance have been observed experimentally either in normal-metal / semiconductor / superconductor junctions [21] or in normal-metal / high- $\mathrm{T}_{c}$ superconductor contacts [22]. Perhaps, the discrete electronic state in our model corresponds to the quantum well state and surface bound state in their situations.

In conclusion, we predict an extraordinary temperature dependence of the resonant AR process in N-QD-S, in which the conductance peak does not exhibit thermal broadening. We also investigate $\mathrm{CBO}$ in N-QD-S, and find that finite temperature induces more resonant peaks around the original $\mathrm{CBO}$ peaks of zero temperature, rather than gives them thermal broadening, which can be applied to determine $\Gamma$ and $\Delta E$ in N-QD-S.

This project was supported by NSFC under Grant No. 10074001. T. H. Lin would also like to thank the support from the Visiting Scholar Foundation of State Key Laboratory for Mesoscopic Physics in Peking University.

* To whom correspondence should be addressed. 


\section{APPENDIX}

In this appendix, we present the atomic solution in equilibrium, which is generalized to the weak coupling case and linear response regime in section II.

In the atomic limit, QD is nearly isolated from the leads, and the negligible coupling to leads determines the equilibrium / non-equilibrium distribution in QD. Suppose QD has multiple discrete energy levels, indexed by $i=1,2, \cdots L$, (here $i$ contains the spin index), and $\mathrm{QD}$ in the atomic limit can be described by

$$
H_{d o t}=\sum_{i=1}^{L} E_{i} c_{i}^{\dagger} c_{i}+U \sum_{i<j} n_{i} n_{j}
$$

in which the second term is the intra-dot Coulomb interaction, $U \equiv e^{2} / 2 C$ is the charging energy of QD.

By using the equation of motion, the retarded Green function of QD can be solved exactly. For example, for a three-level QD, $H_{d o t}=\sum_{i=1}^{3} E_{i} c_{i}^{\dagger} c_{i}+U\left(n_{1} n_{2}+n_{2} n_{3}+n_{3} n_{1}\right)$, one obtains

$$
\left\langle\left\langle c_{1} \mid c_{1}^{\dagger}\right\rangle\right\rangle^{r}=\frac{\left\langle\left(1-n_{2}\right)\left(1-n_{3}\right)\right\rangle}{\omega-E_{1}}+\frac{\left\langle\left(1-n_{2}\right) n_{3}\right\rangle}{\omega-E_{1}-U}+\frac{\left\langle n_{2}\left(1-n_{3}\right)\right\rangle}{\omega-E_{1}-U}+\frac{\left\langle n_{2} n_{3}\right\rangle}{\omega-E_{1}-2 U} .
$$

( $\omega$ contains an infinitesimal imaginary part $\mathrm{i}^{+}$, for all the retarded Green functions). The atomic limit solution have clear physics meaning: due to intra-dot interaction, the single particle energy level $E_{i}$ exhibits several sub-resonances, $E_{i}, E_{i}+U, E_{i}+2 U \cdots$, the weights of these sub-resonances are determined by the occupation configuration of other energy levels. For the three-level QD, $E_{1}$ has the weight of $\left\langle\left(1-n_{2}\right)\left(1-n_{3}\right)\right\rangle$, i.e., the probability of both $E_{2}$ and $E_{3}$ are empty; $E_{1}+U$ has the weights of $\left\langle\left(1-n_{2}\right) n_{3}\right\rangle$ and $\left\langle n_{2}\left(1-n_{3}\right)\right\rangle$, i.e., the

probability of one of $E_{2}$ and $E_{3}$ is occupied and the other empty; $E_{1}+2 U$ has the weight of $\left\langle n_{2} n_{3}\right\rangle$, i.e., the probability of both $E_{2}$ and $E_{3}$ are occupied.

The calculation of the correlation functions like $\left\langle n_{1} n_{2}\right\rangle$ should be done self-consistently when $\mathrm{QD}$ is in the non-equilibrium distribution. However, for the equilibrium QD in the atomic limit, the calculation is straight forward. In this case, the thermal equilibrium QD can be depicted by the density operator $\rho=\frac{1}{Z} e^{-\beta H_{d o t}}$, and the observable $\langle O\rangle$ can be calculated by $\operatorname{Tr}\langle\rho O\rangle$. For the three-level QD, the results are listed as follows 


\begin{tabular}{|l|l|l|l|}
\hline$\left\langle n_{1} n_{2} n_{3}\right\rangle$ & $\frac{1}{Z} e^{-\beta\left(E_{1}+E_{2}+E_{3}+3 U\right)}$ & $\left\langle n_{1}\left(1-n_{2}\right)\left(1-n_{3}\right)\right\rangle$ & $\frac{1}{Z} e^{-\beta E_{1}}$ \\
\hline$\left\langle\left(1-n_{1}\right) n_{2} n_{3}\right\rangle$ & $\frac{1}{Z} e^{-\beta\left(E_{2}+E_{3}+U\right)}$ & $\left\langle\left(1-n_{1}\right) n_{2}\left(1-n_{3}\right)\right\rangle$ & $\frac{1}{Z} e^{-\beta E_{2}}$ \\
\hline$\left\langle n_{1}\left(1-n_{2}\right) n_{3}\right\rangle$ & $\frac{1}{Z} e^{-\beta\left(E_{1}+E_{3}+U\right)}$ & $\left\langle\left(1-n_{1}\right)\left(1-n_{2}\right) n_{3}\right\rangle$ & $\frac{1}{Z} e^{-\beta E_{3}}$ \\
\hline$\left\langle n_{1} n_{2}\left(1-n_{3}\right)\right\rangle$ & $\frac{1}{Z} e^{-\beta\left(E_{1}+E_{2}+U\right)}$ & $\left\langle\left(1-n_{1}\right)\left(1-n_{2}\right)\left(1-n_{3}\right)\right\rangle$ & $\frac{1}{Z} e^{-\beta \cdot 0}$ \\
\hline
\end{tabular}

And the weights in Eq.(A2) can be evaluated with the help of this table, for example, $\left\langle n_{2} n_{3}\right\rangle=\left\langle\left(1-n_{1}\right) n_{2} n_{3}\right\rangle+\left\langle n_{1} n_{2} n_{3}\right\rangle$.

Generally, QD with $L$ energy levels may have $2^{L}$ occupation configurations, represented by $F=\left(N_{1}, N_{2} \cdots N_{L}\right)$ with $N_{i}=0$ or 1 . One has

$$
\begin{aligned}
P(F) & \equiv\left\langle\prod_{i=1}^{L} m_{i}\right\rangle=\frac{1}{Z} e^{-\beta E_{\text {dot }}(F)} \\
m_{i} & \equiv\left\{\begin{array}{cc}
n_{i} & \text { for } N_{i}=1 \\
\left(1-n_{i}\right) & \text { for } N_{i}=0
\end{array},\right.
\end{aligned}
$$

in which $E_{\text {dot }}(F) \equiv \sum_{i} E_{i} N_{i}+U \sum_{i<j} N_{i} N_{j}$ is the total energy of QD for the configuration $F$. Define $\tilde{g}^{r} \equiv \sum_{i=1}^{L}\left\langle\left\langle c_{i} \mid c_{i}^{\dagger}\right\rangle\right\rangle^{r}$, it is easy to obtain

$$
\begin{aligned}
\tilde{g}^{r} & =\sum_{F} P(F) g^{r}(F) \\
g^{r}(F) & =\sum_{i=1}^{L} \frac{1}{\omega-\tilde{E}_{i}(F)},
\end{aligned}
$$

in which $\tilde{E}_{i}(F) \equiv E_{i}+U \sum_{j \neq i} N_{j}$ is the renormalized sing-particle level.

In short, in the atomic limit, the retarded Green function $\tilde{g}^{r}$ is the average over different occupation configurations weighted by a thermal factor, and each configuration behaves as a set of renormalized single particle levels. 


\section{REFERENCES}

[1] A. F. Andreev, Zh. Eksp. Teor. Fiz. 46, 1823 (1964) [Sov. Phys. JETP 19, 1228 (1964)].

[2] G. E. Blonder, M. Tinkham, and T. M. Klapwijk, Phys. Rev. B 25, 4515 (1982).

[3] C. J. Lambert and R. Raimondi, J. Phys.: Condens. Matter 10, 901 (1998).

[4] C. W. J. Beenakker, Phys. Rev. B 46, 12841 (1992).

[5] N. R. Clauphton, M. Leadbeater, and C. J. Lambert, J. Phys.: Condens. Matter 7, 8757 (1995).

[6] Q. -f. Sun, J. Wang, and T. -h. Lin, Phys. Rev. B 59, 3831 (1999).

[7] A. Golub, Phys. Rev. B 54, 3640 (1996).

[8] R. Fazio and R. Raimondi, Phys. Rev. Lett. 80, 2913 (1998).

[9] K. Kang, Phys. Rev. B 58, 9641 (1998).

[10] P. Schwab and R. Raimondi, Phys. Rev. B 59, 1637 (1999).

[11] A. A. Clerk and V. Ambegaokar, Phys. Rev. B 61, 3555 (2000).

[12] A. -P. Jauho, N. S. Wingreen, and Y. Meir, Phys. Rev. B 50, 5528 (1994).

[13] A. L. Yeyati, F. Flores, and A. Martín-Rodero, Phys. Rev. Lett. 83, 600 (1999).

[14] M. A. Kastner, Rev. Mod. Phys. 64, 849 (1992).

[15] Y. Meir, N. S. Wingreen, and P. A. Lee, Phys. Rev. Lett. 66, 3048 (1991).

[16] D. C. Ralph, C. T. Black, and M. Tinkham, Phys. Rev. Lett. 78, 4087 (1997).

[17] S. Guéron et al., Phys. Rev. Lett. 83, 4148 (1999).

[18] A. L. Yeyati et al., Phys. Rev. B 55, R6137 (1997).

[19] H. Takayanagi and T. Akazaki, Phys. Rev. B 52, R8533 (1995).

[20] S. G. den Hartog et al., Phys. Rev. Lett. 77, 4954 (1996).

[21] P. H. C. Magnée et al., Phys. Rev. B 50, 4594 (1994).

[22] Y. Dagan et al., Phys. Rev. B 61, 7012 (2000). 


\section{FIGURE CAPTIONS}

Fig. 1 The zero bias conductance $G$ vs the resonant level $E_{0}$ at different temperature for (a) N-QD-S and (b) N-QD-N. Parameters are: $\Gamma_{L}=\Gamma_{R}=0.1, \Delta=1$ for N-QD-S, $\Delta=0$ for N-QD-N, $k_{B} T=0.001,0.1,0.2,0.3$ corresponding to the decrease of peak heights.

Fig. 2 Schematic picture for understanding the unusual temperature dependence of the conductance in Fig.1. For (a) N-QD-S, resonant AR requires $E_{0}$ lines up with the chemical potential of the superconducting lead. For (b) N-QD-N, single-particle tunneling occurs when the resonant level $E_{0}$ within the range of $k_{B} T$.

Fig. 3 Coulomb blockade oscillations of $G$ vs $V_{g}$ at different temperatures for N-QD-S in Fig.3a-3d and N-QD-N in Fig.3e. QD has two spin degenerate levels, $E_{1 \uparrow}=E_{1 \downarrow}=$ $E_{1}=V_{g}$ and $E_{2 \uparrow}=E_{2 \downarrow}=E_{2}=V_{g}+\Delta E$, with level spacing $\Delta E=0.1$, Coulomb interacting strength $U=1$. Other parameters are: $\Gamma \equiv \Gamma_{1}=\Gamma_{2}=0.002, \Delta=1$ for N-QD-S, $\Delta=0$ for N-QD-N, $k_{B} T=0.01,0.02,0.05,0.10$ marked in the plots. 


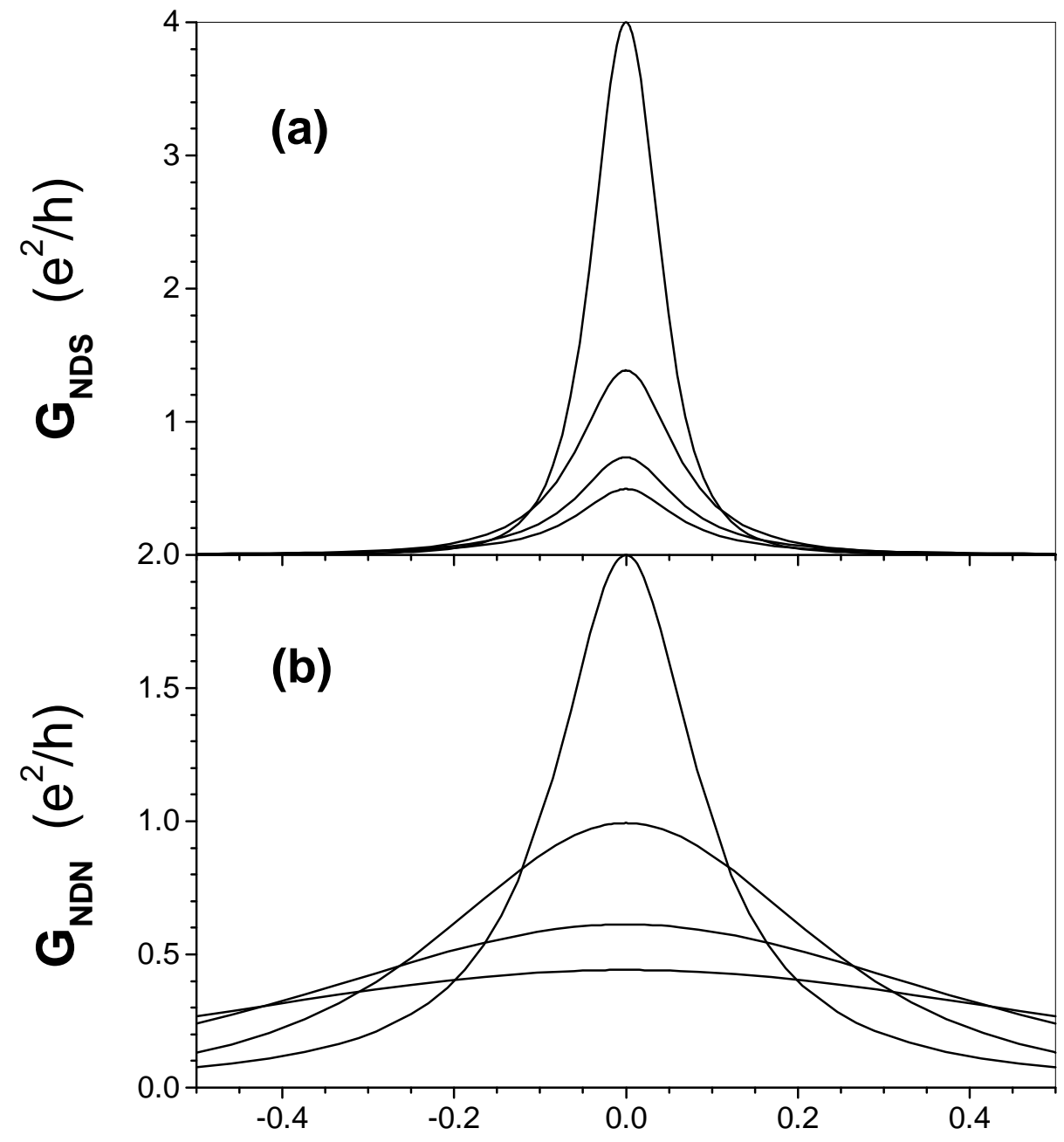

E0

Fig.1 


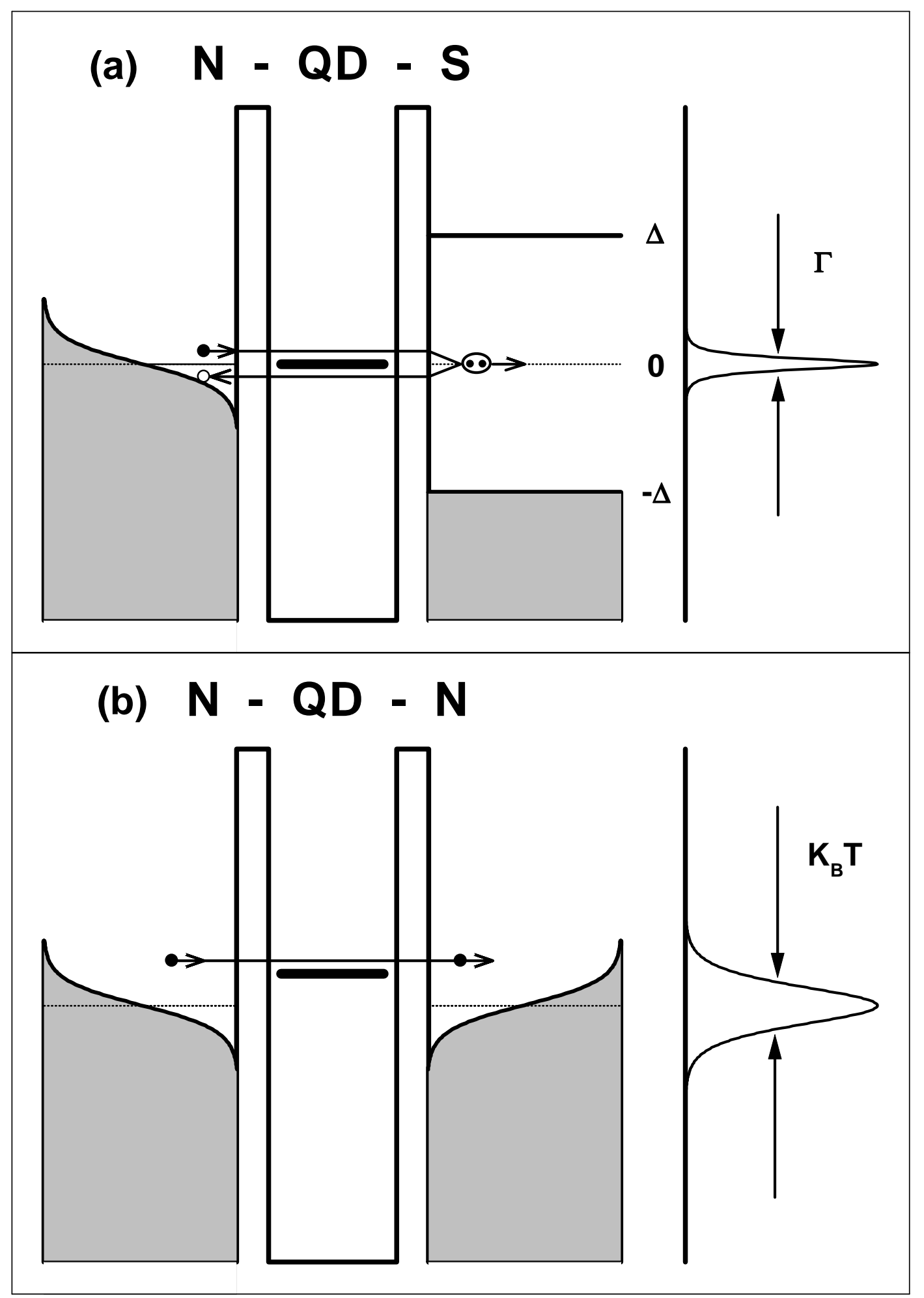

Fig.2 


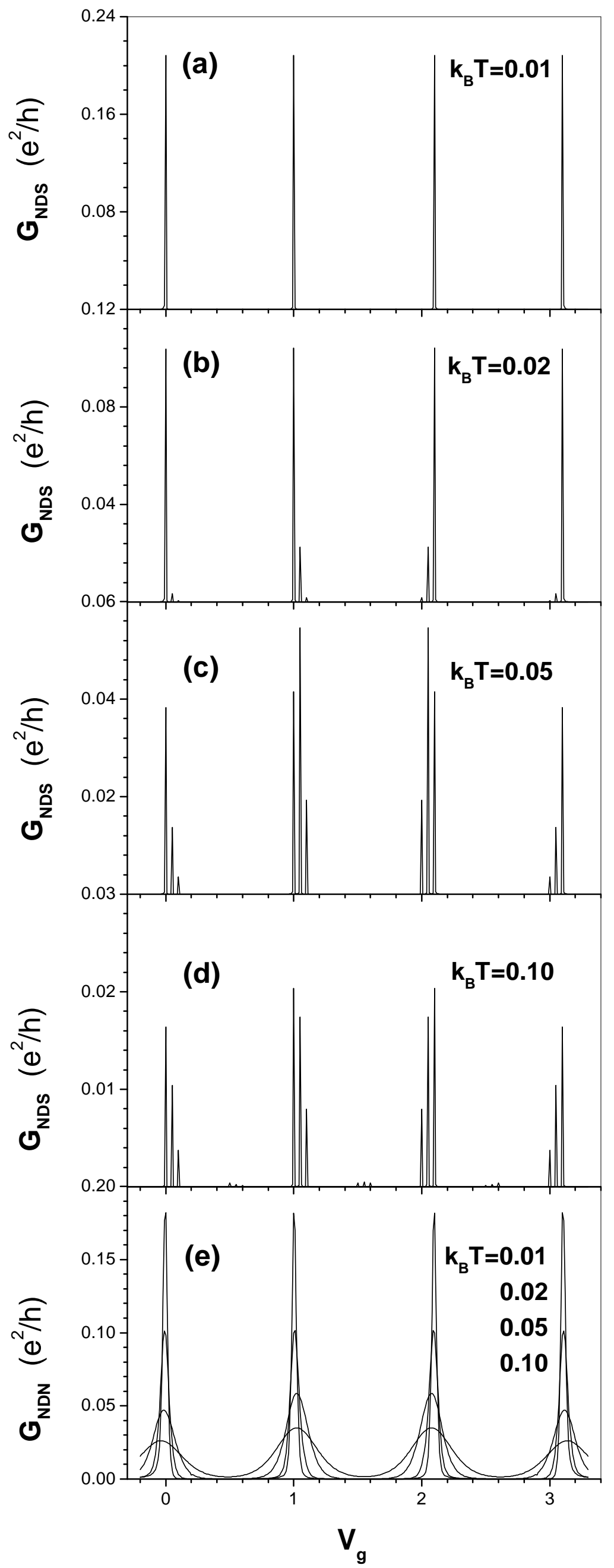

